\title{
AN APPLICATION OF JENSEN'S INEQUALITY IN DETERMINING THE ORDER OF MAGNITUDE OF MULTIPLE FOURIER COEFFICIENTS OF FUNCTIONS OF BOUNDED $\phi$-VARIATION
}

\author{
BHIKHA LILA GHODADRA
}

Abstract. For a Lebesgue integrable complex-valued function $f$ defined over the $n$-dimensional torus $\mathbb{T}^{n}:=[0,2 \pi)^{n}, n \in \mathbb{N}$, let $\hat{f}(\mathbf{k})$ denote the Fourier coefficient of $f$, where $\mathbf{k}=\left(k_{1}, \ldots, k_{n}\right)$ $\in \mathbb{Z}^{n}$. The Riemann-Lebesgue lemma shows that $\hat{f}(\mathbf{k})=o(1)$ as $|\mathbf{k}| \rightarrow 0$ for any $f \in \mathrm{L}^{1}\left(\mathbb{T}^{n}\right)$. However, it is known that, these Fourier coefficients can tend to zero as slowly as we wish. The definitive results are due to V. Fülöp and F. Móricz for functions of bounded variation, and due to B. L. Ghodadra for functions of bounded $p$-variation. In this paper, defining the notion of bounded $\phi$-variation for a function from $[0,2 \pi]^{n}$ to $\mathbb{C}$ in two different ways, we prove that this is the case for Fourier coefficients of such functions also. Interestingly, in proving our main results we use the famous Jensen's inequality for integrals. Our new results with $\phi(x)=x^{p}$ $(p \geqslant 1)$ gives our earlier results [Acta Math. Hungar, 128 (4) (2010), 328-343].

Mathematics subject classification (2010): 42B05, 26D15, $26 \mathrm{~B} 30$.

Keywords and phrases: Jensen's inequality, multiple Fourier coefficient, function of bounded $\phi$-variation in several variables, order of magnitude.

\section{REFERENCES}

[1] C. R. Adams and J. A. Clarkson, Properties of functions $f(x, y)$ of bounded variation, Trans. Amer. Math. Soc., 36, (1934), 711-730.

[2] J. A. Clarkson and C. R. Adams, On definitions of bounded variations for functions of two variables, Trans. Amer. Math. Soc., 35, (1933), 824-854.

[3] B. L. GHOdADRA, Order of magnitude of multiple Fourier coefficients of functions of bounded pvariation, Acta Math. Hungar, 128, 4 (2010), 328-343.

[4] V. FÜLÖP AND F. MóRICZ, Order of magnitude of multiple Fourier coefficients of functions of bounded variation, Acta Math. Hungar, 104, (1-2) (2004), 95-104.

[5] E. W. HobSOn, The theory of functions of a real variable and the theory of Fourier's series, Vol. I, Dover Publications Inc., New York, 1927.

[6] F. MóRICZ, Order of magnitude of double Fourier coefficients of functions of bounded variation, Analysis, 22, (2002), 335-345.

[7] M. SChramm, Functions of $\Phi$-bounded variation and Riemann-Stieltjes integration, Trans. Amer. Math. Soc., 287, (1985), 49-63.

[8] L. C. Young, Sur une generalization de la Notion de variation de puissance p-ieme boranee au sense de M. Wiener, et sur la convergence de series de Fourier, C. R. Acad. Sci. Paris, 204, (1937), 470-472. 